\title{
Sodium and potassium intake and blood pressure change in childhood
}

\author{
Johanna M Geleijnse, Diederick E Grobbee, Albert Hofman
}

\begin{abstract}
Objective-To assess the association between sodium and potassium intake and the rise in blood pressure in childhood.

Design-Longitudinal study of a cohort of children with annual measurements during an average follow up period of seven years.

Setting-Epidemiological survey of the population of a suburban town in western Netherlands.

Subjects-Cohort of 233 children aged 5-17 drawn at random from participants in the population survey.

Main outcome measures - At least six annual timed overnight urine samples were obtained. The mean 24 hour sodium and potassium excretion during the follow up period was estimated for each participant and the sodium to potassium ratio calculated. Individual slopes of blood pressure over time were calculated by linear regression analysis.

Results - No significant association was observed between sodium excretion and the change in blood pressure over time. The mean systolic blood pressure slopes, however, were lower when potassium intake was higher (coefficient of linear regression $-0.045 \mathrm{~mm} \mathrm{Hg} /$ year $/ \mathrm{mmol} ; 95 \%$ confidence interval -0.069 to -0.020 ), and the change in systolic pressure was greater when the urinary sodium to potassium ratio was higher $(0.356 \mathrm{~mm} \mathrm{Hg} /$ year/unit; $95 \%$ confidence interval 0.069 to $0 \cdot 642$ ). In relation to potassium this was interpreted as a rise in blood pressure that was on average $1.0 \mathrm{~mm} \mathrm{Hg}(95 \%$ confidence interval -1.65 to -0.35 ) lower in children in the upper part of the distribution of intake compared with those in the lower part. The mean yearly rise in systolic blood pressure for the group as a whole was $1.95 \mathrm{~mm} \mathrm{Hg}$. Urinary electrolyte excretion was not associated with diastolic blood pressure.
\end{abstract}

Conclusion-Dietary potassium and the dietary sodium to potassium ratio are related to the rise in blood pressure in childhood and may be important in the early pathogenesis of primary hypertension.

\section{Introduction}

Primary hypertension is one of the most important risk factors for cardiovascular disease and probably has its onset in the first decades of life.' Study of blood pressure in childhood may shed light on the aetiology of hypertension and indicate opportunities for measures to prevent hypertension in adult life. Blood pressure in children is determined by genetic and environmental factors. Race, sex, and anthropometric characteristics such as height and body weight have been identified as determinants of blood pressure and the rise in childhood..$^{2+}$ Although the effect of biological maturation is still a subject of debate, alterations in growth and sex hormones may also be related to changes in blood pressure.

The part played by dietary factors has been thorough- ly studied. Sodium intake has been the main topic, but most studies have been concerned with adults. Findings in some but not all interpopulation studies suggest that the prevalence of hypertension is lower in areas with a low sodium intake, although considerable doubt remains about the nature and significance of this. ${ }^{6}$ In children and young adults findings are even less consistent. ${ }^{7}$ Moreover, the rise in blood pressure with age might to some extent be dependent on the average level of sodium intake in a population. Recently the Intersalt Cooperative Research Group found an additional rise in systolic blood pressure of $0.003 \mathrm{~mm}$ $\mathrm{Hg}$ with a sodium intake that was on average $1 \mathrm{mmol} /$ $24 \mathrm{~h}$ higher in adults. ${ }^{8}$ If such an effect of the diet was already acting in childhood and persisted over a lifetime the consequences for the incidence of hypertension in a population might be substantial. ${ }^{4}$ An inverse association of blood pressure with the intake of potassium has been noted occasionally, although this finding remains controversial. ${ }^{10}$ Moreover, there may be an interaction among various dietary electrolytes. In particular, the sodium to potassium ratio may be important.

Because advice on diet may well play a part in intervention, studies on the effect of dietary components on blood pressure remain important. In this study children with high intakes of sodium and potassium were compared with those having low intakes with regard to their individual changes in blood pressure over time. Change in blood pressure rather than actual blood pressure in children may be a predictor of adult blood pressure and of hypertension. ${ }^{112}$

\section{Population and methods}

Between 1975 and 1978 the total population aged 5 years and over in two districts of Zoetermeer, a suburban town in the western part of the Netherlands, was invited to take part in a study of risk factors for cardiovascular disease. ${ }^{3+}$ Of 5670 eligible subjects aged $5-19$ years, $4649(82 \%)$ were examined. From this group a random sample of 596 children was selected for annual follow up in a study of blood pressure tracking and its determinants. ${ }^{3+}$ They were examined four weeks after the initial examination and subsequently at yearly intervals. Children with established secondary hypertension were excluded. For the present analysis we selected children aged up to 17 at entry into the study and whose follow up included at least six yearly examinations. Of the 233 subjects in the cohort, 108 were boys and 125 girls. Their average follow up period was seven years.

\section{Measurements}

Blood pressure measurements were performed with a random zero sphygmomanometer as detailed else- 
where. ${ }^{3}$ Paramedical workers were trained to measure systolic and diastolic blood pressure according to a standardised protocol. Cuffs $23 \mathrm{~cm}$ by 10 or $14 \mathrm{~cm}$ were used, depending on the arm circumference. In children aged over 10 generally the largest cuff was used. Blood pressure was measured in the left arm after 15 minutes' sitting. Diastolic blood pressure was recorded at the fifth Korotkoff phase. Two blood pressure readings were taken and the average used for analysis. Height and body weight were measured with the participant wearing light indoor clothing without shoes. Urine samples were analysed for sodium and potassium concentrations by flame photometry. Urine was collected as six timed overnight samples. ${ }^{131+}$ The collection began at supper and ended with the first urine voided the next morning. Labelled containers were provided, on which the subjects noted the times of starting and finishing each collection. From these the mean 24 hour sodium and potassium intakes were calculated. Electrolyte excretion measured in timed overnight urine samples correlates reasonably well with true mean 24 hour excretion rates in young subjects. ${ }^{1314}$ The main difference between the two

TABLE I - Characteristics of total study group at entry into study

\begin{tabular}{lcc}
\hline & Mean $(\mathrm{SD})$ & Range \\
\hline Age $($ years $)$ & $13 \cdot 2(2 \cdot 7)$ & $5 \cdot 9 \cdot 17 \cdot 0$ \\
Height $(\mathrm{cm})$ & $159 \cdot 3(16 \cdot 2)$ & $115 \cdot 0-193 \cdot 0$ \\
Body weight $(\mathrm{kg})$ & $48 \cdot 8(14 \cdot 6)$ & $20 \cdot 0-80 \cdot 0$ \\
Systolic blood pressure $(\mathrm{mm} \mathrm{Hg})$ & $112 \cdot 4(12 \cdot 9)$ & $81 \cdot 0-153 \cdot 0$ \\
Diastolic blood pressure $(\mathrm{mm} \mathrm{Hg})$ & $68 \cdot 4(8 \cdot 7)$ & $44 \cdot 0-97 \cdot 0$ \\
\hline
\end{tabular}

TABLE II - Yearly rate of change in height, body weight, systolic blood pressure, and diastolic blood pressure during follow up

\begin{tabular}{lc}
\hline & $\begin{array}{c}\text { Rate of change }(95 \% \text { confidence } \\
\text { interval })\end{array}$ \\
\hline Height $(\mathrm{cm} /$ year $)$ & $2 \cdot 61(2 \cdot 31$ to $2 \cdot 91)$ \\
Body weight $(\mathrm{kg} /$ year $)$ & $2 \cdot 85(2 \cdot 60$ to $3 \cdot 10)$ \\
Systolic blood pressure $(\mathrm{mm} \mathrm{Hg} / \mathrm{year})$ & $1 \cdot 95(1.65$ to $2 \cdot 25)$ \\
Diastolic blood pressure $(\mathrm{mm} \mathrm{Hg} /$ year $)$ & $0 \cdot 58(0 \cdot 39$ to $0 \cdot 77)$ \\
\hline$\star^{\text {Coefficient of linear regression. }}$ &
\end{tabular}

TABLE III-Average sodium excretion, potassium excretion, and sodium to potassium ratio, and ranges according to thirds of distributions of electrolyte excretion in study groups during follow up period

\begin{tabular}{|c|c|c|c|}
\hline & \multirow[b]{2}{*}{ Mean } & \multicolumn{2}{|c|}{ Range } \\
\hline & & Lower third & Upper third \\
\hline \multicolumn{4}{|c|}{ Sodium excretion $(\mathrm{mmol} / 24 \mathrm{~h})$ : } \\
\hline Total study group & $135 \cdot 6$ & $61 \cdot 5-117 \cdot 7$ & $147 \cdot 5-251 \cdot 5$ \\
\hline Boys & 140.8 & $61 \cdot 5 \cdot 127 \cdot 2$ & $158 \cdot 2-251 \cdot 5$ \\
\hline Girls & $131 \cdot 1$ & $68 \cdot 5-115 \cdot 0$ & $139 \cdot 8-215 \cdot 3$ \\
\hline Younger group & $138 \cdot 4$ & $68 \cdot 5-119 \cdot 8$ & $147 \cdot 5 \cdot 251 \cdot 5$ \\
\hline Older group & $132 \cdot 8$ & $61 \cdot 5-113 \cdot 5$ & $147 \cdot 5 \cdot 215 \cdot 3$ \\
\hline \multicolumn{4}{|c|}{ Potassium excretion $(\mathrm{mmol} / 24 \mathrm{~h})$ : } \\
\hline Total study group & $43 \cdot 7$ & $15 \cdot 8-37 \cdot 7$ & $47 \cdot 8-77 \cdot 3$ \\
\hline Boys & $47 \cdot 0$ & $20 \cdot 2-41 \cdot 5$ & $51 \cdot 2-77 \cdot 3$ \\
\hline Girls & $40 \cdot 9$ & $15 \cdot 8 \cdot 35 \cdot 3$ & $43 \cdot 5-71 \cdot 0$ \\
\hline Younger group & $43 \cdot 3$ & $15 \cdot 8 \cdot 37 \cdot 2$ & $47 \cdot 3-77 \cdot 3$ \\
\hline $\begin{array}{l}\text { Older group } \\
\text { Ola }\end{array}$ & $44 \cdot 1$ & $20 \cdot 2-38 \cdot 5$ & $47 \cdot 8-75 \cdot 8$ \\
\hline \multicolumn{4}{|c|}{ Sodium to potassium ratio: } \\
\hline Total study group & $3 \cdot 3$ & $1 \cdot 1-2 \cdot 8$ & $3 \cdot 6 \cdot 7 \cdot 4$ \\
\hline Boys & $3 \cdot 1$ & $1 \cdot 1 \cdot 2 \cdot 6$ & $3 \cdot 4 \cdot 7 \cdot 4$ \\
\hline Girls & $3 \cdot 3$ & $1 \cdot 6 \cdot 2 \cdot 8$ & $3 \cdot 7 \cdot 5 \cdot 8$ \\
\hline Younger group & $3 \cdot 3$ & $1 \cdot 6-2 \cdot 8$ & $3 \cdot 6 \cdot 5 \cdot 8$ \\
\hline Older group & $3 \cdot 2$ & $1 \cdot 1-2 \cdot 6$ & $3 \cdot 6-7 \cdot 4$ \\
\hline
\end{tabular}

TABLE IV-Association between vearly rate of change in systolic blood pressure in children and urinary sodium and potassium excretion and urinary sodium to potassium ratio. Findings expressed as regression coefficients ( $\mathrm{mm} \mathrm{Hg/year/mmol} \mathrm{for} \mathrm{sodium} \mathrm{and} \mathrm{potassium;} \mathrm{mm} \mathrm{Hg/year/unit} \mathrm{for} \mathrm{sodium} \mathrm{to} \mathrm{potassium} \mathrm{ratio)}$ for group as a whole and as mean blood pressure slopes in subgroups of children based on thirds of blood pressure distribution ( $\mathrm{mm} \mathrm{Hg} /$ year). ${ }^{\star}$ (95\% Confidence intervals given in parentheses)

\begin{tabular}{|c|c|c|c|}
\hline Blood pressure slope & Sodium & Potassium & Sodium to potassium ratio \\
\hline Regression coefficient & $0.003(\cdots 0.006$ to 0.012$)$ & $-0.045(-0.069$ to -0.020$)$ & $0.356(0.069$ to 0.642$)$ \\
\hline Level in lower third & $2.04(1.56$ to 2.52$)$ & $.44(1.99$ to 2.89$)$ & $1.43(0.97$ to 1.88$)$ \\
\hline Level in middle third & $1.64(1.19$ to 2.09 & $\$ 7(1.42$ to 2.32$)$ & $1.91(1.46$ to 2.36$)$ \\
\hline Level in upper third & $2.12(1.65$ to 2.59 & $: 3(0.98$ to 1.88$)$ & $2.24(1.79$ to 2.69$)$ \\
\hline
\end{tabular}

measurements is a somewhat higher within person variability in the overnight samples. Both methods require multiple samples, and plainly these are more easily collected overnight.

\section{DATA ANALYSIS}

Six complete annual records of each subject were used in the analysis. To quantify the change in blood pressure during the follow up period individual slopes of blood pressure against time were calculated by using linear regression analysis. The association between sodium excretion, potassium excretion, urinary sodium to potassium ratio, and blood pressure slope was analysed by multiple linear regression analysis. To adjust for differences in sex, initial age, and change in height and body weight during follow up these variables were included in the model. When analysing the effect of high sodium intake adjustments were made for potassium intake, and vice versa. Further to assess the effect of electrolyte intake on the change in blood pressure the study group was divided into subgroups based on thirds of the distributions of mean sodium and potassium intake and mean sodium to potassium ratio over the follow up period. In all analyses lower and upper thirds are compared. Data are presented as mean changes per year and $95 \%$ confidence intervals. Equality of group means was tested for both high and low sodium, potassium, and sodium to potassium ratio groups by using analysis of variance. Significance of differences was assessed by two tailed tests throughout. To see whether the relation between electrolyte intake and change in blood pressure was different in boys and girls or in different age groups the data were analysed by sex and age at the initial survey. The median of the initial age distribution was used in stratifying the children for age. In the younger group the age range was $5 \cdot 9-13 \cdot 7$ years and in the older group $13 \cdot 8-17 \cdot 0$ years.

\section{Results}

Table I gives the characteristics of the subjects at the start of the study. Changes in height, body weight, systolic blood pressure, and diastolic blood pressure during the follow up period are given in table II. Table III shows the mean values and ranges of average sodium and potassium excretion and sodium to potassium ratio in the lower and upper thirds of the study groups during the follow up. In boys the mean 24 hour sodium excretion ranged between 61.5 and $251.5 \mathrm{mmol}$, which reflects a daily salt intake of 3.6 $14.7 \mathrm{~g}$. In girls the mean 24 hour sodium excretion ranged between 68.5 and $215.3 \mathrm{mmol}$, corresponding to a salt intake of $4 \cdot 0-12 \cdot 6 \mathrm{~g} / \mathrm{day}$. Potassium intake was higher in boys than in girls and therefore the sodium to potassium ratio was higher in girls. During the study period age showed no independent association with electrolyte intake.

Table IV shows the association between the yearly rate of change in systolic blood pressure and urinary electrolyte excretion. No significant relation between sodium excretion and change in systolic blood pressure could be detected. Urinary potassium excretion, however, was strongly and inversely associated with systolic blood pressure in this cohort (coefficient of linear regression $-0.45 \mathrm{~mm} \mathrm{Hg} / \mathrm{year} / \mathrm{mmol} ; \mathrm{p}=0.0004)$ whereas the systolilc blood pressure slope was higher when the sodium to potassium ratio was higher $(0.356 \mathrm{~mm} \mathrm{Hg} /$ year/unit; $p=0.02)$. The effects of these associations were reflected in the levels of annual increase in systolic blood pressure in children in the lower, middle, and upper thirds of the distribution of electrolyte excretion (table IV).

Figure 1 shows the differences in rise in systolic blood pressure during follow up between subjects with 

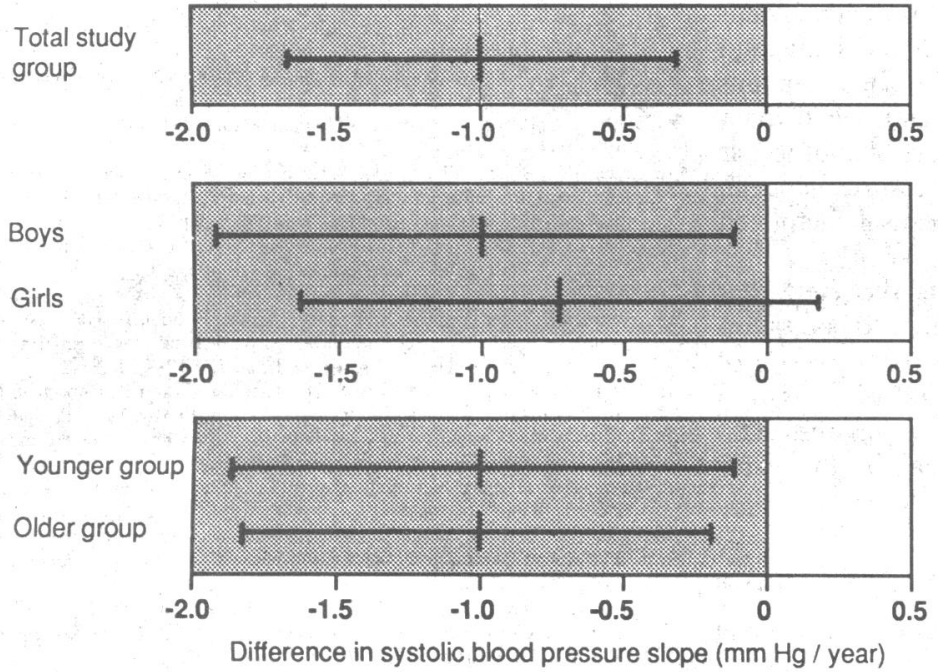

FIG 1 -Differences in yearly rate of change in systolic blood pressure (systolic blood pressure slope $(\mathrm{mm} \mathrm{Hg}$ year)) during follow up between children with high and low potassium intakes. Adjustments made for differences in sex, initial age, change in height and body weight, and sodium intake (mean differences and 95\% confidence intervals)

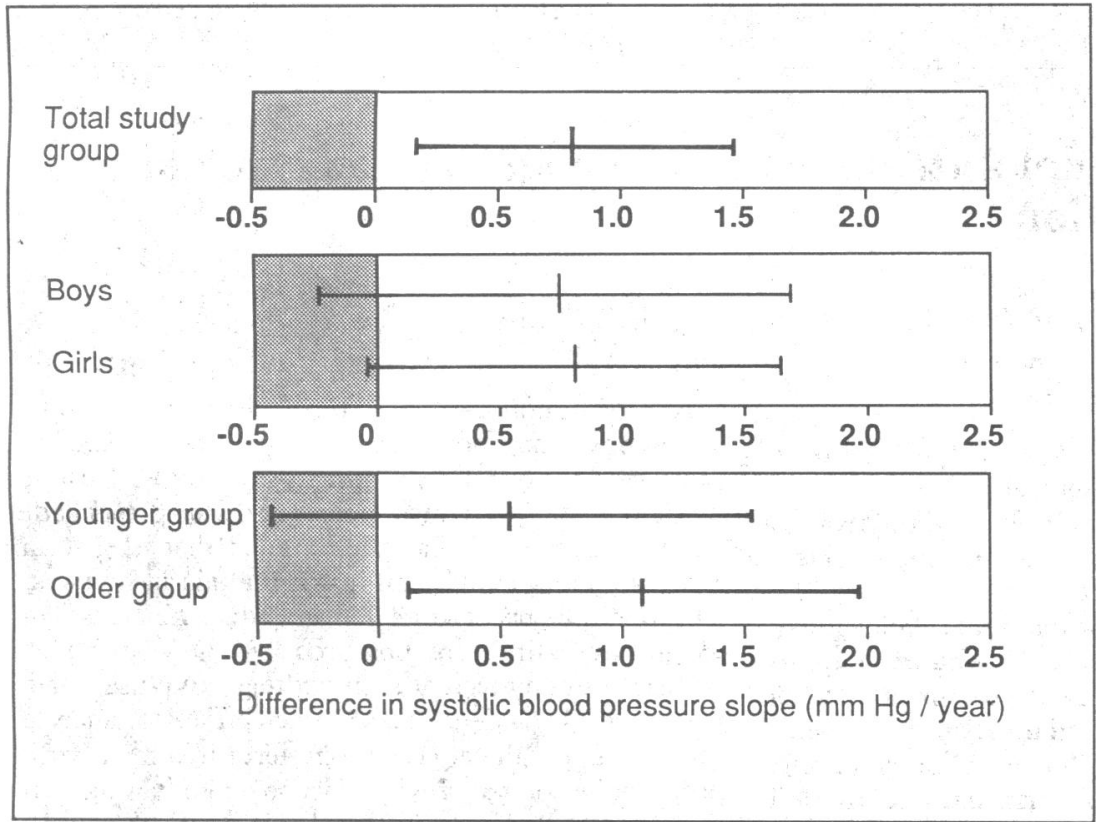

FIG 2-Differences in yearly rate of change in systolic blood pressure (systolic blood pressure slope ( $\mathrm{mm} \mathrm{Hg}$ year)) during follow up between children with high and low urinary sodium to potassium ratios. Adjustments made for differences in sex, initial age, and change in height and body weight (mean differences and $95 \%$ confidence intervals

high and low potassium intakes for the group as a whole and for subgroups based on sex and age. Mean yearly change in systolic blood pressure was $1.4 \mathrm{~mm} \mathrm{Hg}$ in the group with a high potassium intake and $2.4 \mathrm{~mm} \mathrm{Hg}$ in the group with a low potassium intake $(\mathrm{p}=0.007)$. The difference $(1.0 \mathrm{~mm} \mathrm{Hg})$ amounted to half the average yearly change in systolic blood pressure recorded in the group as a whole (table II). This finding seemed to be most pronounced for boys $-2 \cdot 1 \mathrm{~mm} \mathrm{Hg}$ / year in those with a high intake and $3.1 \mathrm{~mm} \mathrm{Hg} /$ year in those with a low intake $(p=0 \cdot 04)$. The stratified analysis disclosed no clear difference in effect in different age groups.

Figure 2 shows the differences in rise in systolic blood pressure between groups with high and low urinary sodium to potassium ratios. In children with a high sodium to potassium ratio a slope of $2.2 \mathrm{~mm} \mathrm{Hg} /$ year was recorded compared with $1.4 \mathrm{~mm} \mathrm{Hg} /$ year in children with a low sodium to potassium ratio $(p=0 \cdot 02)$. The difference in blood pressure slope between groups with high and low ratios was present in both boys and girls. The effect of sodium to potassium ratio on change in systolic blood pressure seemed to be stronger in older children. In the group initially aged $13 \cdot 8-17 \cdot 0$ years the difference between groups with high and low sodium to potassium ratios was $1 \cdot 1 \mathrm{~mm} \mathrm{Hg} /$ year $(\mathrm{p}=0.02)$.

Neither sodium nor potassium nor the sodium to potassium ratio was significantly related to the change in diastolic blood pressure. When the data were reanalysed taking initial blood pressure readings into account results were unaffected.

\section{Discussion}

The main findings in this longitudinal study were that both potassium intake and the ratio of sodium intake to potassium intake were related to the change in systolic blood pressure in childhood. Children with a high dietary intake of potassium had a smaller annual increase in systolic blood pressure than children with a low intake. The sodium to potassium ratio also showed an inverse relation with blood pressure. Interestingly, in this age group there was no clear effect of sodium intake alone. These findings corroborate the report by Lever $e t$ al who hypothesised that in the early stages of hypertension blood pressure is raised by a process related more to potassium than to sodium. ${ }^{\text {is }}$ Moreover, findings in experimental studies suggest that an effect of sodium intake on blood pressure may be present only in older age groups. ${ }^{16}$ One trial conducted in newborn infants, however, found a small but significant effect of sodium intake on the blood pressure slope during the first months of life. ${ }^{1}$

The inverse association between potassium intake and the rise in systolic blood pressure was similar in the two age groups. By contrast, the relation between the sodium to potassium ratio and the blood pressure slope was stronger with age. As this age related phenomenon could not be ascribed to either sodium or potassium it was probably related to an age dependent interaction between the two. Khaw and Barrett-Connor found an increased sensitivity of blood pressure to the dietary sodium to potassium ratio with aging in men. ${ }^{18}$

Some studies have failed to detect a relation between potassium or the sodium to potassium ratio and blood pressure in children or adults. ${ }^{7920}$ This may be explained in several ways. Firstly, in some studies only one urine sample was used to estimate the daily sodium and potassium intake. The large intraindividual variability of urinary cation excretion, notably sodium, may obscure results. ${ }^{21}$ This calls for repeated measurements of electrolyte excretion..$^{14}$ In our study sodium and potassium intakes were assessed by calculating the mean daily sodium and potassium excretion from six timed overnight urine samples. By averaging multiple measurements the effect of intraindividual day to day variability is reduced and sodium and potassium intakes are estimated more precisely. Secondly, if the relation between potassium and blood pressure exists only in childhood or in the early stages of hypertension no observable effect might be present in study populations that are older. ${ }^{15}$ Thirdly, in some surveys on potassium the study period was quite short. If the effect of potassium becomes evident only after a sufficient time of exposure it might be too weak to be detected in these studies.

There are several ways by which potassium intake might affect the rise in blood pressure. ${ }^{22}$ Potassium may reduce blood pressure by vasodilatation, thereby causing a decrease in total peripheral resistance and an increase in cardiac output. Some studies suggest that potassium might lower blood pressure by acting as a diuretic or by altering the activity of the renin-angio- 
tensin system. Others give evidence that potassium might modify peripheral and central neural regulation of blood pressure. We can draw no conclusions from our study about the way that potassium acts. Nor is there a ready explanation for a possible mechanism of sodium-potassium interaction. Finally, there remains a possibility that potassium intake and the urinary sodium to potassium ratio are indicators of another, as yet unknown, determinant of blood pressure change in childhood rather than being direct causal factors in blood pressure regulation.

In conclusion, this study supports the view that dietary potassium and the dietary sodium to potassium ratio may be important in the early pathogenesis of hypertension. Possibly a sufficient intake of potassium or a reduction of the dietary sodium to potassium ratio in youth may prove to be beneficial in the early prevention of hypertension.

1 Hofman A, Grobbee DE, Schalekamp MADH, eds. The early pathogenisis of primary hypertension. Amsterdam: Elsevier, 1987.

2 Akinkugbe $O O$, Akinkugbe FM, Aveni $O$, et al. Biracial study of arterial pressures in the first and second decades of life. Br Med f 1977;i:1132-4.

3 Hofman A, Valkenburg HA. Determinants of change in blood pressure during childhood. Am f Epidemiol 1983;117:735-43.

4 Hofman A, Valkenburg HA. Maas J, Groustra FN. The natural history of blood pressure in children. Int $\mathcal{F}$ Epidemiol 1985;14:91-6.

5 LaBarthe DR, Morris DL, Freer BS. Blood pressure during growth and development. Ann Clin Res 1984;16 (suppl 4):35-43.

6 Simpson FO. Salt and hypertension: current data, attitudes, and policies. f Cardiovasc Pharmacol 1984:6: $44-9$

7 Grobbee DE, Bak AAA. Electrolyte intake and hypertension in children. In
Rettig R, Ganten D, Luft FC, eds. Salt and hypertension. Berlin: Springer Verlag, 1989

8 Intersalt Cooperative Research Group. Intersalt: an international study of electrolyte excretion and blood pressure. Results for 24 hour urinary sodium and potassium excretion. Br. Med f 1988;297:319-28.

9 Hofman A. Blood pressure in childhood: an epidemiologic approach to the aetiology of hypertension. 7 Hypertens 1984;2:323-8.

10 Khaw KT, Thom S. Randomized double-blind crossover trial of potassium on blood pressure in normal subjects. Lancet 1982;ii:1127-9.

11 Szklo M. Epidemiologic patterns of hlood pressure in children. Epidemiol Rez 1979;1:143-69.

12 Lauer RM, Burns TL, Clarke WR. Epidemiology of blood pressure in childhood. In: Hofman A, Grobbee DE, Schalekamp MADH, eds. The early childhood. In: Hofman A, Grobbee DE, Schalekamp MADH, ed
pathogenesis of primary hypertension. Amsterdam: Elsevier, 1987.

13 Liu K, Dyer AR, Cooper RS, Stamler R, Stamler J. Can overnight urine replace 24-hour urine collection to assess salt intake? Hypertension 1979;1 529-36

14 Knuiman JT, van Poppel G, Burema J, van der Heijden I, Hautvast GAJ Multiple overnight urine collections may be used for estimating the excretion of electrolytes and creatinine. Clin Chem 1988;34:135-8.

15 Lever AF, Beretta-Piccoli C, Brown JJ, Davis DL, Fraser R, Robertson JIS Sodium and potassium in essential hypertension. Br Med f 1981:283:463-7. 16 Grobbee DE, Hofman A. Does sodium restriction lower blood pressure? BrMed f 1986:293:27-9.

17 Hofman A, Hazebroek A, Valkenburg HA. A randomized trial of sodium intake and blood pressure in newborn infants. FAMA 1983;250:370-3.

18 Khaw KT, Barrett-Connor E. The association between blood pressure, age, and dietary sodium and porassium: a population study. Circulation 1988;
$77: 53-61$.

19 Frank GC, Bereson GS, Webher LS. Dietary studies and the relationship of diet to cardiovascular disease risk factor variables in 10 -year-old children. The Bogalusa heart study. Am $f$ Clin Nutr 1978;31:328-40.

20 Cooper R, Liu K. Trevisan M, Miller W, Stamler J. Urinary sodium excretion and blood pressure in children: absence of a reproducible association. Hypertension 1983;5:135-9.

21 Liu K, Cooper RS, Soltero I, Stamler J. Variability in 24-hour urine sodium excretion in children. Hypertension 1979;1:631-6.

22 Treasure J, Ploth D. Role of dietary potassium in the treatment of hypertension. Hypertension 1983;5:864-71.

Accepted 3 fanuary 1990)
Department of Public

Health Sciences, Bowman-

Gray School of Medicine,

Winston-Salem, North

Carolina 27103, United

States

Stephan Rössner, MD,

visiting professor

Carol L Taylor, MAS,

statistician

Robert P Byington, PHD,

assistant professor

Curt D Furberg, PHD,

chairman

\section{Correspondence to:}

Dr S Rössner, Obesity Unit,

Karolinska Hospital,

S-104 01 Stockholm,

Sweden.

Br Med f 1990;300:902-3

\title{
Long term propranolol treatment and changes in body weight after myocardial infarction
}

\author{
Stephan Rössner, Carol L Taylor, Robert P Byington, Curt D Furberg
}

\begin{abstract}
Objective-To determine the effect of long term propranolol treatment on body weight.

Design-Retrospective analysis of data from a placebo controlled randomised double blind clinical trial (the $\beta$ blocker heart attack trial).
\end{abstract}

Patients - 3837 Men and women randomised 5-21 days after an acute myocardial infarction to treatment with placebo or propranolol for up to 40 months. Patients were followed up at annual visits.

Main outcome measure-Changes in body weight.

Results - At the first annual visit patients treated with propranolol had gained more weight than those given placebo (mean weight gain $2.3 \mathrm{~kg} v 1.2 \mathrm{~kg}$ respectively, mean difference $1.2 \mathrm{~kg}(95 \%$ confidence interval 0.9 to 1.5 )). These group differences remained at the second and third annual visits. The difference in weight gain could not be explained by discrepancies in the use of diuretics or in physical activity and was similar in patients of both sexes and of all ages.

Conclusion-Long term $\beta$ blockade results in a sustained weight gain.

\section{Introduction}

Recently it has become increasingly clear that obesity is a multifactorial condition. Though the development of obesity implies at least a temporary positive energy balance, factors that explain why some people become overweight and others do not have been surprisingly difficult to identify. A recent study showing that large eaters in fact weighed less than small eaters has underscored the complexity of the problem.
As the autonomic system affects energy metabolism ${ }^{23}$ alterations in autonomic activity might be expected to promote obesity in some human subgroups. Despite numerous studies showing the effects of $\beta$ blockade on thermogenesis it has been argued that long term $\beta$ blockade does not result in weight gain in humans. This might be because small drug induced increases in weight over time are obscured by the continuous increase in body weight seen with age. We used data from the $\beta$ blocker heart attack trial to analyse retrospectively the effect of long term treatment with propranolol compared with placebo on body weight in patients who had had a myocardial infarction.

\section{Patients and methods}

A detailed description of the design of the $\beta$ blocker heart attack trial and its methods has been reported elsewhere. ${ }^{+}$In summary, 3837 men and women who had survived an acute myocardial infarction were randomised within five to 21 days after the infarction to treatment with propranolol or placebo. During an average follow up of 25 months mortality from all causes and fatal and non-fatal myocardial infarctions were significantly reduced.

We analysed height, body weight, and heart rate at baseline and after one, two, and three years of follow up from the trial database by sex, age, concomitant use of diuretics, and reported changes in physical activity. We used a multivariate repeated measures analysis of covariance that adjusted for age, sex, and use of diuretics to determine the possible effect of propranolol on body weight. ${ }^{5}$ As a large proportion of subjects were not followed up for two or three vears several estimation methods were applied, which produced the 